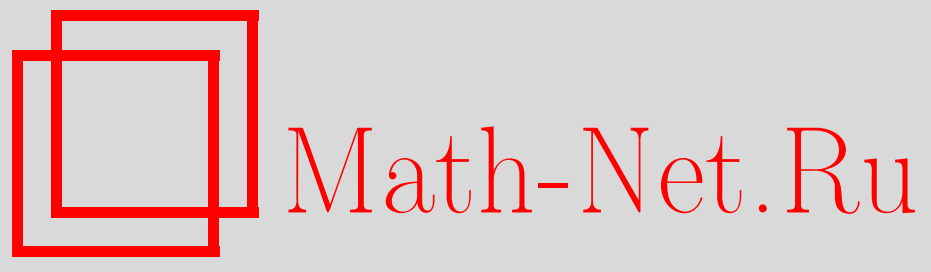

А. А. Кокоткин, О больших подграфах графа расстояний, имеющих маленькое хроматическое число, Матем. заметки, 2014, том 96, выпуск 2, 318-320

Использование Общероссийского математического портала Math-Net.Ru подразумевает, что вы прочитали и согласны с пользовательским соглашением http://www.mathnet.ru/rus/agreement

Параметры загрузки:

IP: 54.197 .130 .99

26 апреля 2023 г., 15:24:00

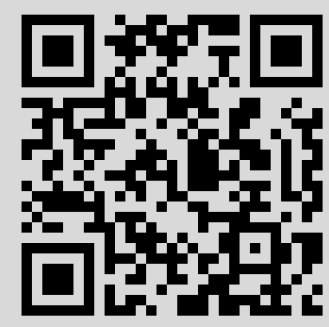




\section{О больших подграфах графа расстояний, имеющих маленькое хроматическое число}

\section{А. А. Кокоткин}

1. Введение и формулировки геометрических результатов. Хроматическое число $\chi\left(\mathbb{R}^{d}\right)$ пространства $\mathbb{R}^{d}$ - это наименьшее количество цветов, в которые можно так покрасить $\mathbb{R}^{d}$, чтобы среди точек одного цвета не нашлось пары точек на расстоянии единица, т.е.

$$
\chi\left(\mathbb{R}^{d}\right)=\min \left\{k \in \mathbb{N}: \exists V_{1}, \ldots, V_{k} \mathbb{R}^{d}=V_{1} \sqcup \cdots \sqcup V_{k}, \forall i \forall \mathbf{x}, \mathbf{y} \in V_{i} \rho(\mathbf{x}, \mathbf{y}) \neq 1\right\},
$$

где $\rho$ - обычное евклидово расстояние.

Легко показать, что для любого $d$ величина $\chi\left(\mathbb{R}^{d}\right)$ конечна. Проблема отыскания хроматического числа пространства была впервые поставлена на рубеже 40-50-х годов XX века (см. [1]-[7]). Несмотря на значительный интерес, вызванный этой проблемой, она до сих пор, по существу, остается нерешенной. Конечно, $\chi\left(\mathbb{R}^{1}\right)=2$, однако уже для плоскости лучшее, что мы знаем, это оценка

$$
4 \leqslant \chi\left(\mathbb{R}^{2}\right) \leqslant 7 .
$$

Для трехмерного пространства мы имеем

$$
6 \leqslant \chi\left(\mathbb{R}^{3}\right) \leqslant 15
$$

(см. [8], [9]), наконец для растущей размерности

$$
(1.239 \cdots+o(1))^{d} \leqslant \chi\left(\mathbb{R}^{d}\right) \leqslant(3+o(1))^{d}
$$

(см. [2], [10]-[12]).

Поставленная задача может быть переформулирована в терминах теории графов. Прежде всего дистаниионным графом (или графом расстояний) назовем конечный граф $G=$ $(V, E)$, вершины которого суть точки евклидова пространства, а ребра соединяют только пары точек, отстоящих друг от друга на расстояние единица. Иными словами,

$$
V \subset \mathbb{R}^{d}, \quad|V|<\infty, \quad E \subseteq\{(\mathbf{x}, \mathbf{y}) \in V \times V: \rho(\mathbf{x}, \mathbf{y})=1\} .
$$

Напомним, что хроматическое число $\chi(G)$ графа $G=(V, E)$ - это наименьшее количество цветов, в которые можно так покрасить его вершины, чтобы вершины одного цвета не соединялись ребром, т.е.

$$
\chi(G)=\min \left\{k \in \mathbb{N}: \exists V_{1}, \ldots, V_{k} V=V_{1} \sqcup \cdots \sqcup V_{k}, \forall i \forall \mathbf{x}, \mathbf{y} \in V_{i} \quad(\mathbf{x}, \mathbf{y}) \notin E\right\} .
$$

Эрдеш и де Брёйн фактически доказали (см. [13]), что $\chi\left(\mathbb{R}^{d}\right)=\max \chi(G)$, где максимум берется по всем графам расстояний в $\mathbb{R}^{d}$. Таким образом, изучение хроматических чисел графов расстояний играет исключительную роль при исследовании проблемы отыскания хроматического числа пространства.

В настоящей работе мы рассматриваем только случай евклидовой плоскости. Тот факт, что $\chi\left(\mathbb{R}^{2}\right) \leqslant 7$, означает, конечно, что для любого графа расстояний $G=(V, E)$ на плоскости $\chi(G) \leqslant 7$. Как следствие, $\alpha(G) \geqslant|V| / 7$, коль скоро через $\alpha(G)$ мы обозначили число

Работа выполнена при поддержке Российского фонда фундаментальных исследований (грант № 12-01-00683).

DOI: $10.4213 / \operatorname{mzm} 5186$ 
независимости графа $G$, т.е. наибольшее количество его вершин, никакие две из которых не соединены ребром:

$$
\alpha(G)=\max \left\{\left|V^{\prime}\right|: V^{\prime} \subset V, \forall \mathbf{x}, \mathbf{y} \in V^{\prime}(\mathbf{x}, \mathbf{y}) \notin E\right\} .
$$

Таким образом, в любом “двумерном" графе расстояний на $n$ вершинах найдется индуцированный подграф, имеющий не менее $n / 7$ вершин и хроматическое число 1 . Это утверждение допускает ряд нетривиальных обобщений и уточнений. Нам удалось доказать следующий результат для графов расстояний на плоскости.

Tеорема 1. Пусть $k \in\{1,2,3,4\}$. В любом графе расстояний $G=(V, E)$ на $n$ вериинах найдется такой индуцированный подграф $G^{\prime}=\left(V^{\prime}, E^{\prime}\right)$, что

$$
\left|V^{\prime}\right| \geqslant\left[\frac{k n}{\varkappa}\right] \quad \text { u } \quad \chi\left(G^{\prime}\right) \leqslant k, \quad \text { әде } \quad \varkappa=4.36 \ldots
$$

Наиболее интересным является случай $k=4$. Фактически он означает, что в каждом графе расстояний на плоскости есть индуцированный подграф, который почти целиком совпадает с исходным графом (содержит не менее $91.7 \%$ его вершин) и допускает раскраску в 4 цвета. Если бы в этом утверждении величину 91.7 удалось заменить на 100, то ввиду теоремы Эрдеша-де Брёйна это бы означало, что $\chi\left(\mathbb{R}^{2}\right)=4$.

\section{2. Постановка вероятностной задачи и формулировки соответствующих ре-} зультатов. Зачастую задачи теории графов допускают нетривиальную интерпретацию в терминах случайного графа. Напомним, что одной из наиболее популярных моделей случайного графа является модель, предложенная Эрдешем и Реньи на рубеже 50-60-х годов XX века (см. [14]). Речь идет о вероятностном пространстве $G(n, p)=\left(\Omega_{n}, \mathscr{B}_{n}, \mathrm{P}_{n}\right)$. Здесь

$$
\Omega_{n}=\{G=(V, E):|V|=n\}
$$

- множество всевозможных графов на $n$ вершинах (без петель и кратных ребер), сигма-алгебра $\mathscr{B}_{n}$ представляет собой множество всех подмножеств $\Omega_{n}$, а

$$
\mathrm{P}_{n}(G)=p^{|E|}(1-p)^{C_{n}^{2}-|E|} .
$$

Иначе говоря, можно считать, что ребра случайного графа появляются независимо друг от друга с вероятностью $p$. Заметим, что в модели Эрдеша-Реньи величина $p$ может зависеть от $n$.

Нас будет интересовать в дальнейшем, с какой вероятностью случайный граф в модели $G(n, p)$ допускает реализацию на плоскости в качестве графа расстояний. Как это часто бывает в науке о случайных графах, при одних значениях $p$ эта вероятность будет стремиться к нулю, а при других - к единице. Определим некоторую критическую величину $p$, отвечающую за вышеупомянутый “фазовый переход”, следующим образом:

$$
p^{*}(n)=\sup \left\{p \in[0,1]: \mathrm{P}_{n}(G \text { реализуется на плоскости как граф расстояний })>\frac{1}{2}\right\} .
$$

Такая величина называется пороговой вероятностъю, и она определена корректно ввиду классических результатов о существовании пороговых вероятностей для монотонных свойств случайных графов (см. [14]).

Мы можем доказать следующие результаты.

Tеорема 2. При $p=c / n$, где $c<1$, выполнено

$$
\mathrm{P}_{n}(G \text { реализуется на плоскости как граф расстояний }) \rightarrow 1, \quad n \rightarrow \infty .
$$


Tеорема 3. При $p=c / n$, где $c>t_{0}=14.797 \ldots$, выполнено

$$
\mathrm{P}_{n}(G \text { реализуется на плоскости как граф расстояний }) \rightarrow 0, \quad n \rightarrow \infty .
$$

Теоремы 2 и 3 означают что

$$
\frac{1-\varepsilon}{n} \leqslant p^{*}(n) \leqslant \frac{t_{0}+\varepsilon}{n}
$$

со сколь угодно малым положительным $\varepsilon$ и $n \geqslant n_{0}(\varepsilon)$. Тем самым, мы знаем порядок роста пороговой вероятности.

\section{СПИСОК ЦИТИРОВАННОЙ ЛИТЕРАТУРЫ}

[1] A. Soifer, The Mathematical Coloring Book. Mathematics of Coloring and the Colorful Life of its Creators, Springer, New York, 2009. [2] А. М. Райгородский, УМH, 56:1 (2001), 107-146. [3] P. Brass, W. Moser, J. Pach, Research Problems in Discrete Geometry, Springer, New York, 2005. [4] A. M. Raigorodskii, "Coloring distance graphs and graphs of diameters", Thirty Essays on Geometric Graph Theory, Springer-Verlag, Berlin, 2013, 429-460. [5] А. М. Райгородский, Матем. сб., 196:1 (2005), 123-156. [6] А. M. Raigorodskii, Combinatorica, 32:1 (2012), 111-123. [7] Е.И. Пономаренко, А. М. Райгородский, Докл. PAH, 454:3 (2014), 268-269. [8] O. Nechushtan, Discrete Math., 256:1-2 (2002), 499-507. [9] D. Coulson, Discrete Math., 256 (2002), 83-90. [10] А. М. Райгородский, УМH, 55:2 (2000), 147-148. [11] А. М. Райгородский, Линейно-алгебраический метод в комбинаторике, МЦНМО, Москва, 2007. [12] D. G. Larman, C. A. Rogers, Mathematika, 19 (1972), 1-24. [13] N. G. de Bruijn, P. Erdős, Nederl. Akad. Wet., Proc., Ser. A, 54:5 (1951), 371-373. [14] B. Bollobás, Random Graphs, Cambridge Stud. Adv. Math., 73, Cambridge Univ. Press, Cambridge, 2001.

\section{А. А. Кокоткин}

институт (государственный университет),

г. Долгопрудный Московской обл.

E-mail: kokocan@yandex.ru 